\title{
Comment on: Gale EAM (2005) Latent autoimmune diabetes in adults: a guide for the perplexed. Diabetologia 48:2195-2199
}

\author{
A. Clark • M. Desai
}

Received: 2 May 2006 / Accepted: 5 June 2006 / Published online: 11 July 2006

(C) Springer-Verlag 2006

To the Editor:

The editorial, 'Latent autoimmune diabetes in adults: a guide for the perplexed' [1] questions the need for the separate classification of autoimmune diabetes developing in adults (latent autoimmune diabetes in adults [LADA]) from the disease developing in childhood (type 1 diabetes). In it Edwin Gale concludes that the evidence is marginal for designation of LADA as a distinct aetiological entity in the burgeoning world of subdivisions of diabetes. We would like to address some of the issues raised with reference to our recent findings in a large cohort of LADA subjects from the UK $[2,3]$. We aim to aid the 'perplexed' in understanding the complexities of LADA.

Approximately $8-12 \%$ patients who are diagnosed over the age of 30 with type 2 diabetes have markers of autoimmunity $[4,5]$, making the prevalence of LADA approximately $50 \%$ of that of classical childhood-onset type 1 diabetes. LADA therefore encompasses a significant proportion of the diabetic population. In an era of genetics, definitions and disease classifications there has been little progress in understanding the aetiology of LADA since its first description in 1977. Although much attention has been paid to assembling names for this heterogeneous group of adult-onset subjects [6], the aetiology remains uncertain and clinical diagnosis rests on autoantibody testing, which provides little instruction for clinical management [1].

\footnotetext{
A. Clark $(\square) \cdot$ M. Desai

Diabetes Research Laboratories, Oxford Centre for Diabetes, Endocrinology and Metabolism, Churchill Hospital, Headington, Oxford OX3 7LJ, UK

e-mail: anne.clark@drl.ox.ac.uk
}

\section{The lines in the sand between changes in beta cell function and/or mass}

One difference between autoimmune diabetes and classical type 2 diabetes is the underlying cause of inadequate insulin secretion. Diminished beta cell mass is the major feature in childhood-onset diabetes; the insulin secretory capacity is usually reduced by $60-80 \%$ by clinical onset. At onset of type 2 diabetes, we believe that the capacity for secretion (beta cell mass) is largely retained but the functional beta cell mass becomes inadequate as a result of defects in beta cell stimulus-secretion coupling. The disease process in LADA could include aspects of reduced mass and diminished functional activity [7]; estimations of beta cell function by modelling, e.g. homeostasis model assessment (HOMA\%B), or from C-peptide secretion imply that there is a surviving (but small) functional population of beta cells at diagnosis in LADA and this can be maintained in some patients for many years after diagnosis $[4,8]$. In addition, treatment regimens to stimulate beta cell function (e.g. sulphonylureas) are effective in many LADA patients [9], although insulin therapy is required more rapidly postdiagnosis than in type 2 diabetic patients [4]. This suggests that a population of functional beta cells can be maintained for variable periods but are eventually lost by autoimmune destructive processes in LADA.

\section{The age-related continuum of the genetic bar code}

The major contribution to genetic susceptibility to childhood-onset autoimmune diabetes is conferred by the HLA region (IDDMI), which provides $50 \%$ of the genetic risk. The younger the age of onset of type 1 diabetes, the greater the genetic load $[10,11]$. As observed in young-onset 
patients, the primary susceptibility HLA haplotypes in LADA are $D R B 1 * 0301-D Q B 1 * 0201$ and $D R B 1 * 0401$ $D Q B 1 * 0302$, and protection is conferred by $D R B 1 * 1501$ $D Q B 1 * 0602$ (M. Desai et al., unpublished observations). As in type 1 diabetes [12], there is an increased frequency of susceptibility genotypes in LADA patients diagnosed at younger ages, and the presence of protective genotypes is attenuated with age (M. Desai et al., unpublished observations). Thus, the contribution of HLA to genetic risk conforms to the same pattern as seen in childhood. Protective and susceptibility alleles could influence not only the timing of the onset of clinically evident autoimmune disease, but also the severity of the clinical course.

The association pattern of insulin gene promoter region polymorphisms (IDDM2) with LADA is also similar to that reported in children [2]; the variable number of tandem repeats (VNTR) class I alleles confer risk, and the class III alleles provide protection. The underlying genetic predisposition from IDDMI and IDDM2 in children and adults is therefore comparable, suggesting that LADA is not a unique disease entity separate from type 1 diabetes. It is likely that other factors, such as the environment, interact with a predisposing genetic background to modify the age of onset and clinical severity of autoimmune disease.

\section{Autoimmunity, antibodies and age}

LADA is defined on the basis of autoantibody positivity; autoantibodies to GAD (GADA) are the most prevalent, whereas autoantibodies to protein tyrosine phosphatase-2 (IA-2) and insulin (IAA) are relatively rare compared with findings in children $[9,13]$. The significance of levels of autoantibodies in relation to the disease process of LADA and their prognostic value are unclear [1]. In the prediagnosis period in children, the combined presence of GADA, IA-2 and/or IAA is associated with more rapid clinical onset of hyperglycaemia than if only one type of antibody were present [14]. Only large and long prospective studies will answer the question of whether autoantibody status pre-diagnosis can predict severity of clinical features in LADA. However, it appears that there is a similar association of multiple antibody positivity at diagnosis in LADA and increased progression of disease, as demonstrated by more rapid requirement for insulin therapy post-diagnosis $[3,13]$.

The question remains whether the autoimmune process of LADA starts in childhood and insulin secretion only becomes inadequate following a precipitating event in adulthood (a very long latency!). In the post-diagnosis period, high and fluctuating levels of GADA persist in LADA (for up to 12 years) [8]; this could imply persistence of antigen in surviving beta cells, sustaining the immune response. However, no relationship exists between GADA levels post-diagnosis and the clinical course of the disease [3]. GADA levels are therefore not helpful predictive markers either for decline in glycaemic control or for requirement for insulin treatment.

\section{Definition, diagnosis and deliberation}

A definition of LADA has recently been issued by the Immunology of Diabetes Society [6]. However, it is unclear how a disease entity with poorly defined aetiology and heterogeneous clinical features can be so rigorously defined; we seem to have returned to definition of diabetes by age ( $>30$ years) and therapy (no insulin requirement within 6 months of diagnosis) and a test for autoantibodies with low positive predictive value for insulin therapy requirement post-diagnosis [1].

In conclusion, there appears to be a continuum with age of both genetic and clinical factors in juvenile- and adultonset autoimmune diabetes; there is a decreased frequency of predisposing HLA variants and diminished severity in clinical course of disease with increasing age of onset in cohorts of children (diagnosed under 25 years) and of adults ( $>30$ years). We agree with the Editor that there is no scientific or clinical evidence to suggest that the overall aetiology of LADA is different from that of childhoodonset autoimmune diabetes. The qualitative differences in clinical features at onset are likely to result from a combination of varying genetic susceptibility and its interaction with the environment. Opinions differ regarding treatment of adult-onset patients, but the UKPDS LADA study shows that the actual therapy received by patients with autoimmune diabetes did not significantly affect the clinical course of the disease. Defining differences on the basis of age of onset is likely to hinder rather than help understanding of autoimmune diabetes so that interventions can be appropriately devised for children and adults alike.

Acknowledgements We thank our colleagues in OCDEM for helpful discussions and the Wellcome Trust (A. Clark) and Diabetes UK (M. Desai) for funding.

\section{References}

1. Gale EAM (2005) Latent autoimmune diabetes in adults: a guide for the perplexed. Diabetologia 48:2195-2199

2. Desai M, Zeggini E, Horton VA et al (2006) The variable number of tandem repeats upstream of the insulin gene is a susceptibility locus for latent autoimmune diabetes in adults. Diabetes 55:1890-1894

3. Clark A, Desai M, Cull CA et al (2005) Relationship of autoantibodies to glutamic acid decarboxylase (GADA) to deterioration of glycaemic control assessed by therapy progression in latent autoimmune diabetes in adults (LADA) in the UKPDS. Diabetologia 48(Suppl 1):A86 
4. Turner R, Stratton I, Horton V et al (1997) UKPDS 25: autoantibodies to islet-cell cytoplasm and glutamic acid decarboxylase for prediction of insulin requirement in type 2 diabetes. UK Prospective Diabetes Study Group. Lancet 350:1288-1293

5. Tuomi T, Carlsson A, Li H et al (1999) Clinical and genetic characteristics of type 2 diabetes with and without GAD antibodies. Diabetes 48:150-157

6. Fourlanos S, Dotta F, Greenbaum CJ et al (2005) Latent autoimmune diabetes in adults (LADA) should be less latent. Diabetologia 48:2206-2212

7. Borg H, Gottsater A, Landin-Olsson M, Fernlund P, Sundkvist G (2001) High levels of antigen-specific islet antibodies predict future beta-cell failure in patients with onset of diabetes in adult age. J Clin Endocrinol Metab 86:3032-3038

8. Borg H, Gottsater A, Fernlund P, Sundkvist G (2002) A 12-year prospective study of the relationship between islet antibodies and beta-cell function at and after the diagnosis in patients with adultonset diabetes. Diabetes 51:1754-1762

9. Davis TM, Wright AD, Mehta ZM et al (2005) Islet autoantibodies in clinically diagnosed type 2 diabetes: prevalence and relationship with metabolic control (UKPDS 70). Diabetologia 48:695-702

10. Caillat-Zucman S, Garchon HJ, Timsit J et al (1992) Agedependent HLA genetic heterogeneity of type 1 insulin-dependent diabetes mellitus. J Clin Invest 90:2242-2250

11. Gillespie KM, Gale EAM, Bingley PJ (2002) High familial risk and genetic susceptibility in early onset childhood diabetes. Diabetes 51:210-214

12. Graham J, Kockum I, Sanjeevi CB et al (1999) Negative association between type 1 diabetes and HLA DQB1*0602DQA $1 * 0102$ is attenuated with age at onset. Swedish Childhood Diabetes Study Group. Eur J Immunogenet 26: $117-127$

13. Bottazzo GF, Bosi E, Cull CA et al (2005) IA-2 antibody prevalence and risk assessment of early insulin requirement in subjects presenting with type 2 diabetes (UKPDS 71). Diabetologia 48:703-708

14. Bingley PJ, Bonifacio E, Williams AJ et al (1997) Prediction of IDDM in the general population: strategies based on combinations of autoantibody markers. Diabetes 46:1701-1710 